\title{
Modeling and Optimization of High-Sensitivity, Low-Volume Microfluidic-Based Surface Immunoassays
}

\author{
Martin Zimmermann, ${ }^{1, *}$ Emmanuel Delamarche, \\ Marc Wolf, ${ }^{1}$ and Patrick Hunziker ${ }^{1}$ \\ ${ }^{1}$ University Hospital Basel, Petersgraben 4, 4031 Basel, Switzerland \\ ${ }^{2}$ IBM Research GmbH, Zürich Research Laboratory, Säumerstrasse 4, \\ 8803 Rüschlikon, Switzerland \\ E-mail: martin.zimmermann@unibas.ch
}

\begin{abstract}
Microfluidics are emerging as a promising technology for miniaturizing biological assays for applications in diagnostics and research in life sciences because they enable the parallel analysis of multiple analytes with economy of samples and in short time. We have previously developed microfluidic networks for surface immunoassays where antibodies that are immobilized on one wall of a microchannel capture analytes flowing in the microchannel. This technology is capable of detecting analytes with picomolar sensitivity and from sub-microliter volume of sample within $45 \mathrm{~min}$. This paper presents the theoretical modeling of these immunoassays where a finite difference algorithm is applied to delineate the role of the transport of analyte molecules in the microchannel (convection and diffusion), the kinetics of binding between the analyte and the capture antibodies, and the surface density of the capture antibody on the assay. The model shows that assays can be greatly optimized by varying the flow velocity of the solution of analyte in the microchannels. The model also shows how much the analyte-antibody binding constant and the surface density of the capture antibodies influence the performance of the assay. We then derive strategies to optimize assays toward maximal sensitivity, minimal sample volume requirement or fast performance, which we think will allow further development of microfluidic networks for immunoassay applications.
\end{abstract}

Key Words. microfluidic, surface immunoassay, lab-on-a-chip, highsensitivity, low-volume

\section{Introduction}

Microfluidics and biochips, which are derived from microfabrication techniques, are emerging as powerful bioanalytical platforms (Tay, 2002; Ekins, 1998). Microfluidics in particular, have the potential to detect multiple analytes in a small volume of sample and can integrate multiple functionalities for the processing of samples and the generation and acquisition of signals (Manz and Becker, 1998). For these reasons, microfluidic devices are developed and used for detecting disease markers and screening drug candidates as well as for antibody characterization (Nguyen and Wereley, 2002). Different methods can be employed to move liquids in microfluidics, such as pressure-driven flow, electro-osmosis, or acceleration. Ideal microfluidics are simple to utilize, reliable, fast, sensitive, versatile, and cheap. One of the simplest approaches for producing flow in capillaries is to use capillary forces. Capillary-driven flow requires no peripheral equipment, and this concept is used for portable immunodiagnostic tests (Apple et al., 1999).

We are interested in developing capillary-driven microfluidic chips for diagnostic and bioanalytical applications in which fluorescence surface immunoassays are performed in the microchannels of the chip, samples of one microliter in volume or less are used, multiple analytes are detected, and sensitivities down to $1 \mathrm{pM}$ concentration of analytes can be achieved. An important step in developing these devices was to employ a hydrophobic elastomeric substrate for the assay and covering the elastomer with a Si microfabricated element having a plurality of microchannels (Bernard et al., 2001b). The assay takes place on the areas of the elastomer that are exposed to the microchannels. Each microchannel starts with a loading pad and ends with a capillary pump (Juncker et al., 2002). The capillary pressure along the flow path is encoded to secure the unidirectional filling of the liquid from the loading pad to the capillary pump. Therefore, assays are done by loading the necessary reagents (e.g. capture antibody and detection antibody) and samples in the correct sequence in each loading pad. The assay conditions in adjacent microchannels can be varied independently because the elastomer seals the microchannels efficiently. This concept was recently exemplified by detecting TNF$\alpha$ in $0.6 \mu \mathrm{L}$ volume of cell culture supernatant with $1 \mathrm{pM}$ sensitivity, and up to 170 test sites were made on a $1 \mathrm{~mm}^{2}$ area of the elastomer (Cesaro-Tadic et al., 2004). This concept was also qualitatively applied to detect disease markers such as C-reactive protein (Wolf et al., 2004). Because clinically relevant analyte concentrations are often spread over a wide range from micromolar to picomolar concentrations, optimizing the microfluidic devices in terms of covering a large dynamic range for the measurements is important. At the same time, a microfluidic device should also be suited to handle very small concentrations and if possible minute volumes of analyte.

\footnotetext{
${ }^{*}$ Corresponding author.
} 
We are interested in examining these previous results analytically to identify the predominant assay parameters and to derive optimization strategies for performing assays with such microfluidics for having optimal sensitivity, minimal sample requirement, or fastest possible times to results.

Like other computational studies on the kinetics of antigen binding in microchannels (Vijayendran et al., 1999; Myszka et al., 1998; Edwards, 1999; Edwards et al., 1999; Jenkins et al., 2004), our computational model of fluid dynamics includes convection, diffusion and receptor-ligand kinetics (Sapsford et al., 2001; Balgi et al., 1995) of capillary-flow-driven microfluidic channels and was implemented using a finite difference approach (Griebel et al., 1995). Thereby, we solved the steady-state uncompressed Navier-Stokes equation, combined with a convection-diffusion model and a fluid-wall interaction model which we describe below.

\section{Methods}

\subsection{Microfluidic networks used in the analyses}

Our model is implemented to describe the binding of an antigen to a surface-immobilized capture antibody in a microchannel, Figure 1, and is based on the geometrical characteristics of microfluidic networks that we used previously (Cesaro-Tadic et al., 2004). The microfluidic networks had several independent microchannels, and we therefore only need to consider one microchannel. Each microfluidic channel is $20 \mu \mathrm{m}$ deep, $30 \mu \mathrm{m}$ wide and $1 \mathrm{~mm}$ long. In typical experiments, the capture antibodies are present in the second half of the channel, and the analytes cannot be lost by sticking to the walls elsewhere than at the capture sites because the walls are treated to be proteinrepellent (Prime and Whitesides, 1991). The capture area is covered by individual capture antibody molecules with

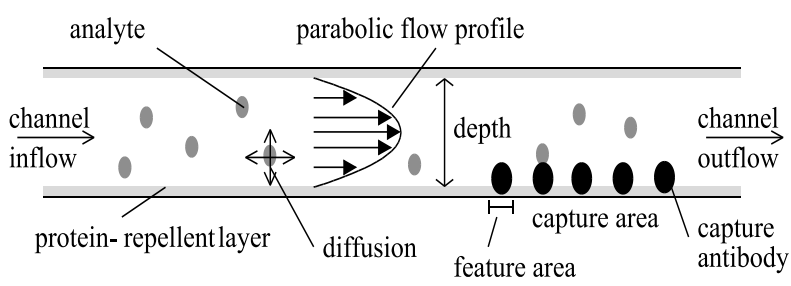

Fig. 1. Configuration of a surface immunoassay in a microfluidic channel. The liquid enters the channel at the inlet on the left-hand side and flows through the channel under laminar conditions with a parabolic flow profile. The flowing analyte solution contains molecules which are transported in the channel. The molecules are allowed to diffuse perpendicularly to the flow direction and can be captured by the capture antibodies. Wall elements not covered with capture antibodies are protected against unspecific binding, which in the simulation is regarded as perfect. a given surface density. We define the "feature area" as the total capture area divided by the number of active capture antibodies. If all capture antibodies were properly oriented and able to bind two antigens each, the feature area would be equal to half the footprint of a capture antibody. In practice, only a fraction of the capture antibodies might be functional and we will therefore explore the influence of the feature area on the assays.

The analyte solution flows into the microchannel at constant flow velocity and flow rate. The flow of an aqueous solution inside a small channel is typically laminar (Reynolds number $\ll 1$ ) and has a parabolic flow profile. The analyte molecules are transported by convection along the flow direction and in addition are free to diffuse. Each time an analyte molecule reaches a capture site, a standard ligand-receptor binding model is applied to define whether capture occurs. Both the association and dissociation events are modeled. The binding kinetics as well as the convection-diffusion model are described in more detail in the next section.

In the model, we define the analyte exploitation as the ratio between the number of captured analyte molecules and the total number of analyte molecules that were present in a given volume of sample that passed over the capture zone. An assay where all analyte molecules would flow in the microchannel without being captured would have an analyte exploitation of zero and would generate no signal. Inversely, an ideal assay would have an analyte exploitation of one. The available volume of analyte solution is considered to be unlimited except when the simulation is used to study the exploitation of analyte.

\subsection{Models}

The flow of a liquid in a region over time $t$ is characterized by a velocity vector field $\vec{u}$, a pressure $p$ and a density $\rho$. For laminar, incompressible and viscous fluids the density is constant. The flow is described by the Navier-Stokes partial differential equation system

$$
\begin{aligned}
\frac{\partial}{\partial t} \vec{u}+(\vec{u} \cdot \operatorname{grad}) \vec{u}+\operatorname{grad} p & =\frac{1}{R e} \Delta \vec{u}+\vec{g} \\
\operatorname{div} \vec{u} & =0
\end{aligned}
$$

in dimensionless form with the Reynolds number $R e$ and external forces $\vec{g}$. External forces such as gravity can be neglected in such miniaturized systems. For $R e \ll 2100$, flow is considered to be laminar and has a characteristic parabolic flow profile with zero flow velocity at the channel walls and peak flow velocity in the channel center. Here, $R e$ is $\sim 0.07$ for the maximum flow rates considered.

The bulk concentration $C$ of a solute in a given solution is described by the Convection-Diffusion equation of the 
form

$$
\frac{\partial C}{\partial t}+\vec{u} \cdot \operatorname{grad} C=D \Delta C+\Theta(t, x, y, C)
$$

with a diffusion coefficient $D$, a source term $\Theta$ and the identical velocity vector field $\vec{u}$ given in equation (1). We have applied the Stokes-Einstein-relation $D=\frac{k T}{6 \pi \eta R_{\mathrm{h}}}$, with the hydrodynamic radius $R_{\mathrm{h}}$, the analyte viscosity $\eta$ and the Boltzmann constant $k$ to estimate the diffusion coefficient $D$ of the analyte molecule to $D=10^{-6} \mathrm{~cm}^{2} \mathrm{~s}^{-1}$ which we used for all further calculations and which corresponds to the literature (Metsämuuronen et al., 2002) where comparable values for small molecules such as TNF- $\alpha$ are reported. The analyte viscosity was set to a high plasma viscosity (Koenig et al., 1998) of $2 \mathrm{mPa}$.

The association and the dissociation from the capture site are described by the rate coefficients $k$, the analyte concentration $C$ and the density of free binding sites $\left(\Theta_{\max }-\Theta_{t}\right)$ on the surface, using an ordinary differential equation of the form,

$$
\frac{d \Theta_{\mathrm{t}}}{d t}=k_{\mathrm{on}} C\left(\Theta_{\max }-\Theta_{\mathrm{t}}\right)-k_{\text {off }} \Theta_{\mathrm{t}}
$$

for monovalent receptors and ligands. $k_{\text {on }}$ is the rate constant for association, $k_{\text {off }}$ is the rate constant for dissociation, $C$ is the concentration of free molecules in the fluid, $\Theta_{\mathrm{t}}$ is the surface density at time $t . \Theta_{\max }$ is the maximum surface density of molecules calculated from the feature area of the individual capture molecules and is assumed to be constant over time. In this simulation we generally used $10^{6} \mathrm{M}^{-1} \mathrm{~s}^{-1}$ for $k_{\mathrm{on}}$ and $10^{-3} \mathrm{~s}^{-1}$ for $k_{\text {off }}$ (Santora et al., 2001), but in some case these constants were modified.

The set of equations (1)-(4) is used to implement the model describing the capture of an analyte by a surfaceimmobilized capture site in a microfluidic channel. This model uses the finite difference approach to calculate the influences of the key parameters on the capture of analyte. The model is detailed in the Supplementary Information, Part A, and validated in Part B by comparing results from the simulation with analytical results when the equations could be solved analytically. Equation (4) in particular can be solved analytically for a constant analyte concentration:

$$
\Theta_{\mathrm{t}}=\frac{k_{\mathrm{on}} \Theta_{\mathrm{max}} C}{k_{\mathrm{on}} C+k_{\mathrm{off}}}\left(1-e^{-\left(k_{\mathrm{on}} C+k_{\mathrm{off}}\right) t}\right) .
$$

The maximum binding density $\hat{\Theta}_{\text {bound }}$ of analyte on the capture area depends only on the analyte concentration and the binding constants $k_{\text {on }}$ and $k_{\text {off }}$ which are also known as the equilibrium constant $K$ :

$$
\hat{\Theta}_{\text {bound }}=\frac{k_{\mathrm{on}} \Theta_{\mathrm{max}} C}{k_{\mathrm{on}} C+k_{\mathrm{off}}}
$$

$$
\begin{aligned}
K & =\frac{k_{\mathrm{on}}}{k_{\mathrm{off}}} \\
{[K] } & =\frac{\left[\mathrm{s}^{-1} \mathrm{M}^{-1}\right]}{\left[\mathrm{s}^{-1}\right]} .
\end{aligned}
$$

\section{Results}

The combination of equations (1) to (4) allows the study of the influence of flow rate, analyte bulk concentration, capture-site surface density and binding-rate coefficients on the binding kinetics. Experimentally, captured analytes might be detected directly using surface-sensitive techniques or by binding them with fluorescently-labeled detection antibodies. We do not need to take the binding between captured analytes and detection antibodies into account because this step is generally not limiting in immunoassays: a high concentration of detection antibodies can be used to ensure fast completion of this binding reaction, for example. First, we need to determine when the capture reaction is reaction-limited or transport-limited.

\subsection{Reaction-limited versus transport-limited kinetics of capture}

One of the key parameters that determine whether the kinetics of capture of analyte is reaction-limited or transportlimited is the velocity of the analyte solution in the microchannel. The capture of the analyte is reaction-limited if the number of molecules binding to the capture site per unit of time remains similar for different flow velocities. In contrast, the capture of the analyte is transport-limited if the number of analyte molecules binding per unit of time is sensitive to the flow velocity of the sample in the microchannel. The analyte exploitation is represented in Figure 2 as a function of the flow velocity. It increases with decreasing flow velocity because insufficient mass transport increases the reaction time for capture. For velocities above $0.5 \mathrm{~mm} \mathrm{~s}^{-1}$, corresponding to flow rates higher than $18 \mathrm{~nL} \mathrm{~min}^{-1}$ for the channels used in previous experiments, a system with a binding constant $K$ of $10^{9} \mathrm{M}^{-1}$ and a feature area of $1500 \mathrm{~nm}^{2}$ is reactionlimited: the residency time of the analytes on the capture area is short and the analyte exploitation therefore is small $(\sim 5 \%)$. At lower flow rates, the analyte exploitation increases but the binding reaction becomes transportlimited because lower volumes of analyte solution are available at the capture area per unit of time and thus fewer molecules have a chance to be captured per unit of time. A flow velocity of $0.7 \mathrm{~mm} \mathrm{~s}^{-1}$ was used in previous work (Cesaro-Tadic et al., 2004), and we suggest that these experiments were performed in the reaction-limited regime. We consider this flow velocity as well as lower flow velocities in the remainder of the paper to encompass 


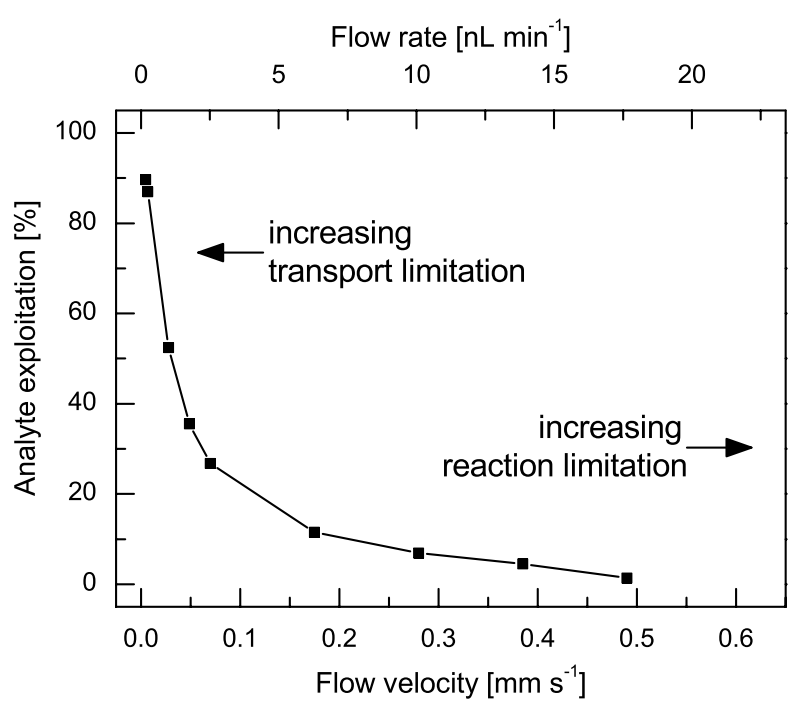

Fig. 2. Calculated mean analyte exploitation as a function of the flow velocity in the first five minutes of the incubation time with $1 \mathrm{pM}$ analyte concentration. Adjusting the flow velocity (and flow rate) affects the exploitation of the analyte molecules in a sample and is therefore useful to minimize the absolute volume of analyte required for saturation of the capture sites. The line is provided as a guide to the eye. A binding constant of $10^{9} \mathrm{M}^{-1}$, analyte concentration of $1 \mathrm{pM}$ and a feature area of $1500 \mathrm{~nm}^{2}$ were selected here.

the different regimes. We specifically choose a flow velocity of $0.07 \mathrm{~mm} \mathrm{~s}^{-1}$, where transport limitation already occurs, and a flow velocity of $0.007 \mathrm{~mm} \mathrm{~s}^{-1}$, where the capture according to Figure 2 falls in the transport-limited regime. In this latter case, longer incubation times are necessary to reach an equivalent signal intensity to the reaction-limited case. In contrast to the transport-limited case, the molecules in the sample volume are used more efficiently.

We investigate the influence of the flow velocity and the feature area on the number of analytes captured per unit area, Figure 3. Capture antibodies that are passively adsorbed on a hydrophobic surface have a footprint of $\sim 150 \mathrm{~nm}^{2}$ (Delamarche, 2004; Amzel and Poljak, 1979) and it has been suggested that for this mode of deposition $\sim 10 \%$ of the antibodies might be correctly oriented and preserved for capturing one antigen from solution (Wild, 2001), which here would correspond to a feature area of $1500 \mathrm{~nm}^{2}$. Using oriented antibodies and advanced surface-immobilization strategies, the feature area can be decreased. We consider for this reason a scenario where each capture antibody has a chance to bind one antigen (feature area of $150 \mathrm{~nm}^{2}$ ). The model developed here can also be applied to situations with very different feature areas, microchannel dimensions, binding constants, and flow velocities.

Figure 3 shows how many analyte molecules are captured per unit area as a function of time for feature areas of
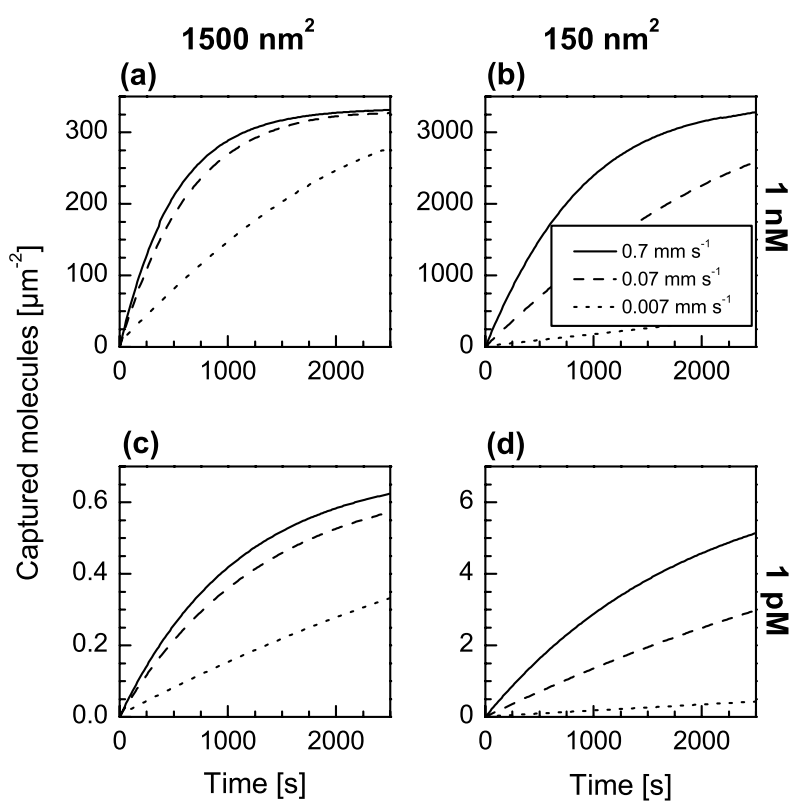

(d)

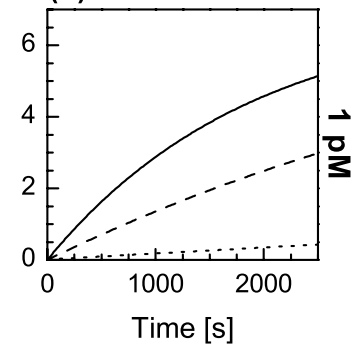

Fig. 3. Binding of analytes in solution to surface-immobilized antibodies as a function of time and for a concentration of analyte of $1 \mathrm{nM}(a, b)$ and $1 \mathrm{pM}(c, d)$, and for a feature area of $1500 \mathrm{~nm}^{2}(a, c)$ and $150 \mathrm{~nm}^{2}(b, d)$. This binding reaction corresponds to the capture step of a surface immunoassay and takes place in a microchannel as shown in Figure 1. The binding reaction is more sensitive to mass transport limitations for the smaller feature areas ( $b$ and $d$ ) as well as for lower analyte concentrations ( $c$ and d). A hypothetical analyte-antibody binding constant of $K=10^{9} \mathrm{M}^{-1}$ was chosen for these analyzes.

1500 and $150 \mathrm{~nm}^{2}$, various flow rates, and analyte concentrations of $1 \mathrm{nM}$ and $1 \mathrm{pM}$. We selected $1 \mathrm{nM}$ for the analyte concentration because it corresponds to immunoassays that are relatively sensitive and used for many clinical applications (Wild, 2001). A concentration of 1 pM represents a practical limit in sensitivity for immunoassays and we want to use the model developed here to optimize assays done using microfluidics for this analyte concentration. Figure 3(a) and (b) show that, as expected, the maximum surface density of captured analyte is inversely proportional to the feature area, but also that the kinetics of capture is affected by the feature area. For a larger feature area $\left(1500 \mathrm{~nm}^{2}\right)$, it is less important to keep the flow velocity high enough to repopulate the microchannel with new analytes because the assay is reaction-limited. This explains the similar outcomes of assays done using flow velocities of 0.7 and $0.07 \mathrm{~mm} \mathrm{~s}^{-1}$. The binding reaction becomes transport-limited for a flow velocity of $0.007 \mathrm{~mm} \mathrm{~s}^{-1}$, as visible in Figure 3(a). The graphs in Figure 3(b) indicate that capture zones having a smaller feature area exploit the solution from analyte faster than those with a larger feature area. As a consequence, the flow rate has a larger influence on the binding kinetics in the case of smaller feature areas. 
The surface density of captured analytes decreases dramatically when analytes are at $1 \mathrm{pM}$ concentration, Figure 3(c) and (d), which is dictated by the equilibrium constant $K$. The binding reaction is slower than at $1 \mathrm{nM}$ in all cases, and the flow velocity as well as the feature area affect the capture reaction in a similar way as at the higher concentration.

\subsection{Linear binding saturation curves at very low flow velocities}

Biological assays that need a long time to reach equilibrium might have to be stopped early for convenience. The graphs in Figure 3(a) and (b) show that saturation in binding is nearly reached within $1000 \mathrm{~s}$ for a flow velocity of $0.7 \mathrm{~mm} \mathrm{~s}^{-1}$. The binding reactions for lower flow velocities are much slower and show a linear behavior. Although assays done under these conditions yield small signals, they can be conveniently stopped at any point in time, because uncertainties on the incubation time give small errors on the signal. The model predicts that an assay with $1500 \mathrm{~nm}^{2}$ feature area, $1 \mathrm{nM}$ analyte concentration, and $0.007 \mathrm{~mm} \mathrm{~s}^{-1}$ flow velocity has a relative error of only $\pm 4 \%$ when the incubation time is $300 \pm 15$ s. Interestingly, such an assay leads to having $52 \pm 2$ captured antigen per $\mu \mathrm{m}^{2}$, which after the binding of detection antibodies should correspond to a signal well above the limit of detection of conventional fluorescence scanners. The model suggests that assays done with a $1 \mathrm{nM}$ analyte concentration and different conditions have a similar relative error when they are stopped after an incubation time of $300 \pm 15$ s. The graphs in Figure 3(c) and (d) reveal a similar linear evolution of the signal with time for a $1 \mathrm{pM}$ analyte concentration. However, stopping the assay after an arbitrarily short time for this lower analyte concentration is unlikely to be practical because of the much lower surface density of captured analyte after $300 \mathrm{~s}$. Long incubation times are therefore crucial for assays with $1 \mathrm{pM}$ analyte concentration.

\subsection{Influence of the feature area on the binding kinetics}

The density of active capture sites in surface immunoassays usually plays an important role in defining the maximum number of analyte molecules that can be captured and therefore the maximum signal surface density that can be reached. Preventing the denaturation of capture antibodies during and after their deposition is therefore important, and methods to orient antibodies so as to have their epitope-binding part exposed at the surface-liquid interface are sometimes employed. As explained above, we based most of our modeling on feature areas of 1500 and $150 \mathrm{~nm}^{2}$, but now also investigate the role of the feature area on the efficiency of the assay for $1 \mathrm{nM}$ and $1 \mathrm{pM}$

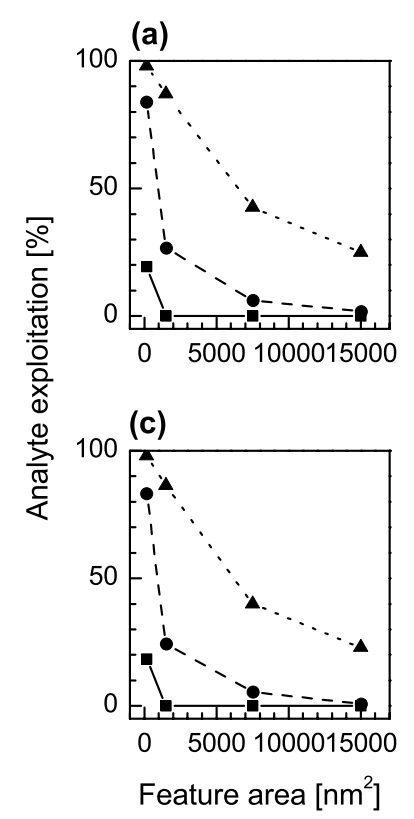

(b)

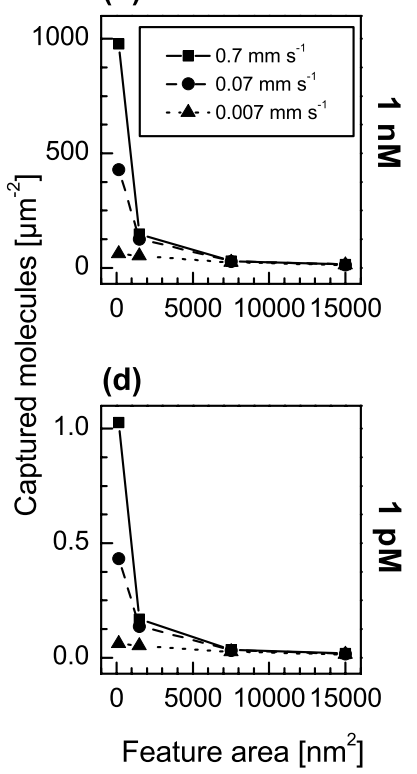

Fig. 4. Efficiency of the capture of analyte molecules flowing at various velocities in a microchannel as shown in Figure 1 as a function of feature area and analyte concentration and for the first 5 min of the assay. The ratio between analyte molecules flowing in the microchannel and analyte molecules captured defines the analyte exploitation $(a, c)$ and the resulting density of analyte molecules captured on the surface $(b, d)$. The lines are provided as guides to the eye.

analyte concentration in more detail, Figure 4. We are in particular interested in learning how the feature area affects assays that have critical requirements such as utilizing a very small amount of sample or being fast. We therefore modeled the analyte exploitation for 5-min-long assays using the same flow rates as before. As expected, all graphs in Figure 4 show that the surface-density of captured analytes increases with the inverse of the feature area so that the exploitation of analyte is maximal with a feature area of $150 \mathrm{~nm}^{2}$. The capture and analyte exploitation are particularly sensitive to the feature area for flow velocities $\leq 0.07 \mathrm{~mm} \mathrm{~s}^{-1}$. The binding constants $k_{\text {on }}$ and $k_{\text {off }}$ in equation (5) enforce the same time to equilibrium for different surface densities of the capture area: the number of capture events per unit of time is larger for surfaces having a higher density of capture sites but the maximum number of capture events is also proportionally larger on these surfaces. As an example, an assay where the feature area is $150 \mathrm{~nm}^{2}$ (first data points) is able to capture $\sim 20 \%$ of the analyte molecules when the analyte solution has a concentration of $1 \mathrm{nM}$ and a flow velocity of $0.7 \mathrm{~mm} \mathrm{~s}^{-1}$, Figure 4(a). This corresponds to an analyte exploitation of $20 \%$. The analyte exploitation falls off dramatically if the feature area is $\geq 1500 \mathrm{~nm}^{2}$. Reducing the flow velocity will, however, increase the analyte exploitation significantly. The analyte exploitation is $\sim 86 \%$ 
when the feature area is $1500 \mathrm{~nm}^{2}$ (second data points) and the flow velocity $0.007 \mathrm{~mm} \mathrm{~s}^{-1}$, for example.

The signal in the assay is proportional to the surface density of captured analytes, and we therefore need to consider what the effect of the feature area on the number of analytes captured per unit area is, Figure 4(b). For feature areas $\leq 1500 \mathrm{~nm}^{2}$, the density of captured molecules is sensitive to the flow velocity and maximum for a velocity of $0.7 \mathrm{~mm} \mathrm{~s}^{-1}$. We learned from Figure 4(b) that for these conditions the analyte exploitation is poor, but the overall number of analyte captured is the highest. The capture step of the assay here is clearly transport-limited. For feature areas $\geq 7500 \mathrm{~nm}^{2}$, the number of captured molecules is small and not sensitive to the flow velocity. Comparing the signals obtained for different flow velocities in the transport-limited regime, a slow velocity of $0.007 \mathrm{~mm} \mathrm{~s}^{-1}$ gives a very small signal compared with that for a velocity of 0.07 or $0.7 \mathrm{~mm} \mathrm{~s}^{-1}$. Assays done with an analyte concentration of $1 \mathrm{pM}$ exhibit a similar behavior as those with $1 \mathrm{nM}$ analyte concentration, Figure 4(c) and (d), except that the density of captured molecules is always $\sim 1000$ times smaller.

We have so far analyzed the performance of the assays done in a microchannel for two key analyte concentrations, and will next explore in more detail how the analyte concentration affects the analyte exploitation, the density of analytes captured on the surface, and the time needed to reach equilibrium. We will also evaluate the influence of the binding constant on the assay.

\subsection{Effect of the analyte concentration on the analyte exploitation}

Both the analyte concentration and the binding constant $K$ determine the maximum density of analytes captured on the surface at equilibrium. Equation (6) shows that a maximum of $0.1 \%$ of the capture sites can bind an analyte at equilibrium when the analyte concentration is $1 \mathrm{pM}$ and the binding constant $10^{9} \mathrm{M}^{-1}$. This ratio increases to $50 \%$ for an analyte concentration of $1 \mathrm{nM}$. We take an arbitrary duration of $5 \mathrm{~min}$ for the capture step, flow velocities of $0.7,0.07$ and $0.007 \mathrm{~mm} \mathrm{~s}^{-1}$, and a $1500 \mathrm{~nm}^{2}$ feature area to investigate how the analyte exploitation and the density of analytes captured on the surface vary with the concentration, Figure 5. At analyte concentrations $\leq 1 \mathrm{nM}$, the analyte exploitation is nearly invariant with the concentration but is strongly influenced by the flow velocity, as already discussed in Figure 2. This situation changes as soon as the analyte concentration becomes larger than $K$. The analyte exploitation is an important criterion for optimizing the assay when the total number of analyte molecules in a sample is strongly limited, whereas it becomes unimportant for high analyte concentration, Figure 5. Five minutes suffice to have nearly all capture

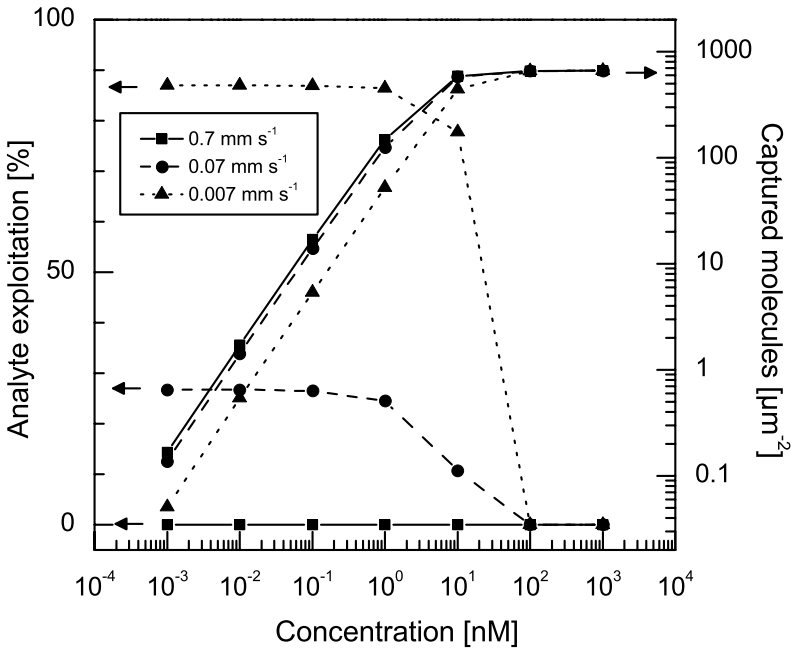

Fig. 5. Mean analyte exploitation and surface density of captured analytes resulting from a 5-min-long assay for various flow velocities and analyte concentrations. The binding constant chosen here was $K=10^{9} \mathrm{M}^{-1}$. The lines are provided as guides to the eye.

sites bound to an analyte when the analyte concentration is $\sim 10$ times larger than the equilibrium constant. Below this concentration value, the surface density of captured molecules diminishes strongly with the concentration of analyte, as expected from equation (5). It becomes helpful in this case to employ a flow velocity $\geq 0.07 \mathrm{~mm} \mathrm{~s}^{-1}$ to minimize transport limitation. Importantly, the model predicts that there will only be $\sim 0.16$ captured analyte per $\mu \mathrm{m}^{2}$ for a 5-min-long assay using a flow velocity of $0.7 \mathrm{~mm} \mathrm{~s}^{-1}$ and an initial analyte concentration of $1 \mathrm{pM}$. Assays for such a low analyte concentration must be longer and if possible should have a smaller flow velocity. Figure 3(d) showed that a sensitivity of $1 \mathrm{pM}$ can be achieved using an incubation time of $\sim 2500$ s for the capture, which is consistent with previous experimental work (CesaroTadic et al., 2004).

We now evaluate how strongly high concentrations of analyte affect the time needed to saturate the capture sites, Figure 6. We know from Figure 5 that saturation of the capture sites can be reached for analyte concentrations $\geq 10 \mathrm{nM}$. With analyte concentrations $\geq 100 \mathrm{nM}$, the capture sites are saturated within a few seconds. An assay to detect an analyte at $100 \mathrm{nM}$ concentration, using a $1500 \mathrm{~nm}^{2}$ feature area and a flow velocity of $0.7-$ $0.07 \mathrm{~mm} \mathrm{~s}^{-1}$, leads to a maximum signal after $45 \mathrm{~s}$, according to our model. This illustrates well the advantage of miniaturizing assays using a microfluidic system. It is preferable to reach equilibrium when the analyte concentration is high, as otherwise variations in the capture duration render the assay imprecise. Conventional assays prevent signal saturation and imprecision due to variations of the incubation time by using a dilution series of the 


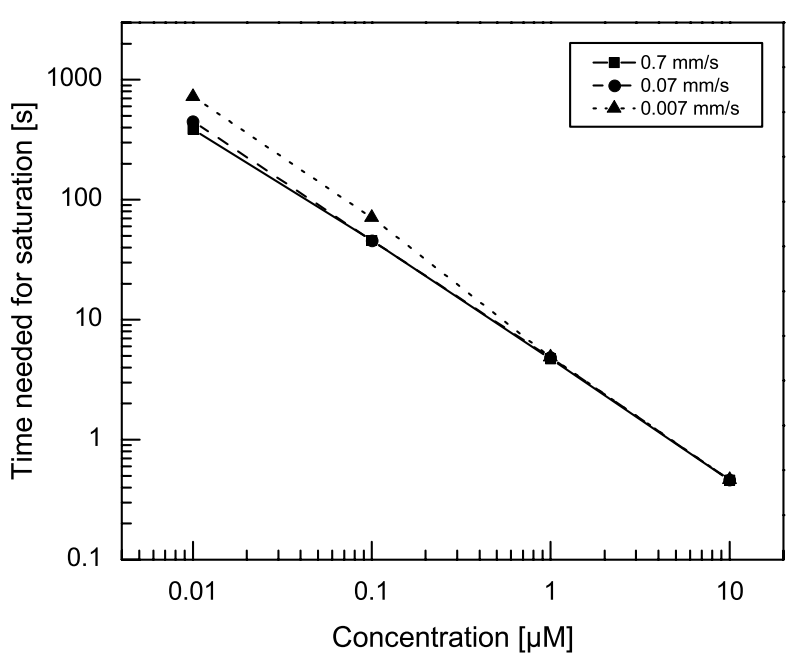

Fig. 6. Time to complete saturation of the capture sites as function of analyte concentration. At concentrations $\leq 1 \mu \mathrm{M}$, time to equilibrium can be increased by reducing the flow velocity and the feature area. For analyte concentrations above $10 \mu \mathrm{M}$, the capture sites are saturated in less than $1 \mathrm{~s}$. The lines are provided as guides to the eye.

sample. The model shows a relative insensitivity of the assay to the flow velocity for concentrations $\geq 1 \mu \mathrm{M}$. The assays are in this case clearly reaction-limited.

\subsection{Influence of the binding constant on the binding kinetics}

The binding constant $K$ between the antigen and the capture antibody plays an important role on the maximum surface density of analyte molecules that can be captured at equilibrium. We have seen above that the assay is very sensitive to the analyte concentration when the concentration falls below $K$. As we study the time response of the binding, we need to distinguish the different influences of the association and dissociation constants. The key factors giving an antibody high or low affinity are the association constant $k_{\text {on }}$ and the dissociation constant $k_{\text {off }}$. The influence of $K$ was therefore investigated for a 5 -min-long capture step by setting $k_{\text {on }}$ from $10^{4}$ to $10^{8} \mathrm{M}^{-1} \mathrm{~s}^{-1}$ while keeping $k_{\text {off }}$ unchanged and vice versa. Figure 7(a) shows that the analyte exploitation increases toward $100 \%$ with increasing $k_{\text {on }}$, and the effect of $k_{\text {on }}$ on the analyte exploitation convolves with the flow rate. A significant analyte exploitation can be observed even for a high flow velocity of $0.7 \mathrm{~mm} \mathrm{~s}^{-1}$ provided that $k_{\text {on }}$ is very high $\left(10^{8} \mathrm{M}^{-1} \mathrm{~s}^{-1}\right)$. Figure 7 (b) shows that when $k_{\text {off }}$ is varied and $K<10^{9} \mathrm{M}^{-1}$, the analyte exploitation is limited because having $k_{\text {off }}>0.001 \mathrm{~s}^{-1}$ results in relatively high dissociation rates. For $K \geq 10^{9} \mathrm{M}^{-1}$ the association constant dominates the binding reaction, and the analyte exploitation is only sensitive to the flow velocity. (a)

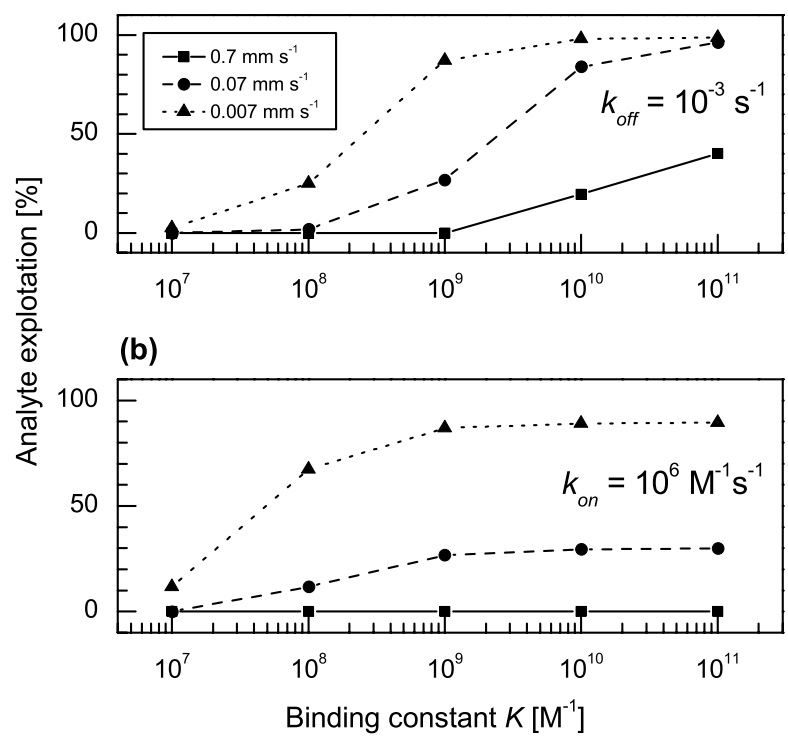

Fig. 7. Mean exploitation of analyte volume as function of the binding constant in the first 5 min of the reaction. An increasing association constant $k_{\mathrm{on}}(a)$ results in increased exploitation of analyte volume, whereas modification of the dissociation rate $k_{\mathrm{off}}(b)$ affects the exploitation of a $1 \mathrm{pM}$ analyte only for $K<10^{9} \mathrm{M}^{-1}$ using a feature area of $1500 \mathrm{~nm}^{2}$. The lines are provided as guides to the eye.

\subsection{Comprehensive assay optimizations}

The detailed model developed above enables us to propose several strategies to optimize surface assays in which capture antibodies are present on one wall of a microchannel and analytes have a concentration of only $1 \mathrm{pM}$. We keep the geometry of the microchannel $\left(30 \times 20 \mu \mathrm{m}^{2}\right.$ in cross section), the capture area (500 $\mu \mathrm{m}$ in length), and the binding constant $K$ at $10^{9} \mathrm{M}^{-1}\left(k_{\text {on }}=10^{6} \mathrm{M}^{-1} \mathrm{~s}^{-1}\right.$ and $k_{\text {off }}=$ $10^{-3} \mathrm{~s}^{-1}$ ) the same as in the previous sections. In the following discussion we consider that the minimum surface density of captured molecules that can be detected using a conventional fluorescence scanner is $\sim 0.5$ analyte per $\mu \mathrm{m}^{2}$ (Genetix, 2004). The remaining parameters influencing the capture step are the flow velocity (or flow rate), the feature area and the time for the capture step, all of which are varied in the next simulations. On a practical level, the flow rate can be changed by varying the evaporation rate of filled capillary pumps or by changing the depth of the channels.

3.6.1. Optimizing assays for fast measurement. We are interested in exploring how fast a sensitive (analyte concentration $\leq 1 \mathrm{nM}$ ) sandwich fluorescence immunoassay can be. The two important steps in such an assay are the capture of analyte from solution and the binding of fluorescently-labeled detection antibodies to the captured analyte. The capture is likely to be the limiting step 
because detection antibodies are usually provided at high concentrations $\left(\mu \mathrm{g} \mathrm{mL}^{-1}\right)$. We therefore focus on the capture step as it was the case in the sections above. First, we assume that the available volume of sample does not limit the assay (e.g. volume $>10 \mu \mathrm{L}$ ), and second, we arbitrarily set the duration of the capture step to $120 \mathrm{~s}$ for an analyte concentration of either $1 \mathrm{nM}$ or $1 \mathrm{pM}$. This duration is $\sim 12$ times shorter than the capture time used for experiments in which TNF- $\alpha$ was detected with a $1 \mathrm{pM}$ sensitivity (Cesaro-Tadic et al., 2004). To obtain a sufficient signal in such a short time, the binding reaction must be very efficient and fast, especially for low analyte concentration where only a small fraction of the capture sites can be bound with analytes at equilibrium. A crucial strategy for fast assays is then to have a high flow velocity to prevent mass transport limitations and a small feature area to increase the analyte exploitation. Small feature areas might be achieved by orienting the capture antibody on the surface and/or roughening the surface used for capture. Figure 8 predicts the surface density of analytes captured on the surface for 150 and $1500 \mathrm{~nm}^{2}$ feature areas. The model suggests that generating a strong enough signal in only $120 \mathrm{~s}$ is not challenging when the analyte has a concentration of $1 \mathrm{nM}$, Figure 8(a) and (b). The density of surface-bound analytes should in this case amount to 422 per $\mu \mathrm{m}^{2}$. A high flow velocity of $0.7 \mathrm{~mm} \mathrm{~s}^{-1}$ is beneficial in the case of small feature areas. At $1 \mathrm{pM}$ analyte concentration, the limit of detection cannot be reached in $120 \mathrm{~s}$ if the feature area is $1500 \mathrm{~nm}^{2}$, Figure 8(c). A smaller feature area is helpful to increase the surface density of captured analytes close to the detection threshold if the
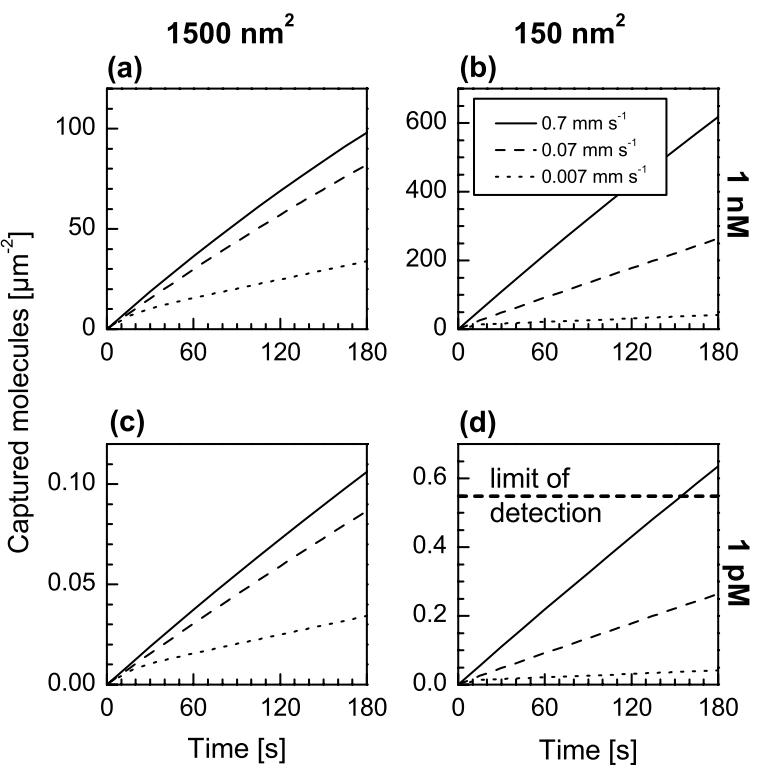

(d)

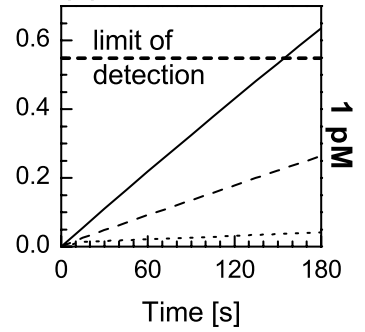

Fig. 8. Number of captured molecules per $\mu m^{2}$ as a function of time for an analyte concentration in (a), (b) [(c), (d)] of $1 \mathrm{nM}[1 \mathrm{pM}]$ and a feature area in $(a),(c)[(b),(d)]$ of $1500 \mathrm{~nm}^{2}\left[150 \mathrm{~nm}^{2}\right]$. flow velocity is $0.7 \mathrm{~mm} \mathrm{~s}^{-1}$, Figure $8(\mathrm{~d})$. If the flow velocity is further increased to $2.8 \mathrm{~mm} \mathrm{~s}^{-1}$ (data not shown), the model predicts that an analyte concentration down to $1 \mathrm{pM}$ can be measured in $120 \mathrm{~s}$, yielding a surface density of captured analytes of $0.68 \mu \mathrm{m}^{-2}$.

3.6.2. Optimizing assays using minimal volumes of analyte. For some applications, only very limited amounts of sample analyte might be available and having a fast assay is less important. We now investigate what the smallest volume of analyte solution needed to detect an analyte at a concentration of $1 \mathrm{pM}$ is. In this case, the analyte has to be exploited as much as possible.

We learned from Figure 4(c) that an exploitation of the analyte of $>95 \%$ can be achieved by using a feature area of $150 \mathrm{~nm}^{2}$ and a flow velocity of $0.007 \mathrm{~mm} \mathrm{~s}^{-1}$ (flow rate of $0.25 \mathrm{~nL} \mathrm{~min}{ }^{-1}$ ). With this feature area and flow velocity, the surface density of captured analytes becomes sufficient for detection after an incubation time of $55 \mathrm{~min}$. This translates into a consumption of only $\sim 13.8 \mathrm{~nL}$ of sample. If the feature area is $1500 \mathrm{~nm}^{2}$ and the flow velocity is $0.007 \mathrm{~mm} \mathrm{~s}^{-1}$, the analyte exploitation is $\sim 86 \%$ and the incubation to achieve sufficient signal increases to $\sim 85 \mathrm{~min}$. In this case, $\sim 21.3 \mathrm{~nL}$ of sample is necessary. A higher signal might be desirable and we therefore consider the case where the surface density of captured analytes is 2 per $\mu \mathrm{m}^{2}$. The volume of analyte needed with a $1 \mathrm{pM}$ analyte concentration and a flow velocity of $0.7 \mathrm{~mm} \mathrm{~s}^{-1}$ becomes $\sim 263 \mathrm{~nL}$ and the assay will take $10.5 \mathrm{~min}$. A slower flow of $0.07 \mathrm{~mm} \mathrm{~s}^{-1}$ would increase the analyte exploitation and require a slightly longer assay and much less sample: $25.5 \mathrm{~min}$ and $\sim 63.8 \mathrm{~nL}$, respectively.

3.6.3. Increasing the dynamic range of the assay. Many biological assays have a limited dynamic range due to the equilibrium behavior dictated by equation (5). Saturation of the capture sites is easily reached for concentrations of analyte (e.g. $100 \mathrm{nM}$ ) that are higher than $\frac{1}{K}$, and the surface density of captured analytes diminishes dramatically as soon as the analyte concentration becomes smaller than $\frac{1}{K}$. Sensitive assays will be limited in dynamic range mostly by saturation at which high analyte concentrations lead to a fast and complete coverage of all binding sites, which makes it impossible to distinguish between different elevated concentrations of analyte. The dilution of the analyte sample that solves this problem with assays done using microtiter plates is not an ideal solution for a microfluidic-based immunoassay. We suggest that flushing a solution of analyte through an array of parallel microchannels at different flow rates 
can expand the dynamic range of the assay. Figure 6 suggested that decreasing the flow velocity from 0.7 to $0.007 \mathrm{~mm} \mathrm{~s}^{-1}$ will increase the time needed to saturate the capture sites with a $100 \mathrm{nM}$ analyte concentration from 45 to $70 \mathrm{~s}$. The freedom in designing microchannels of various heights and depths in Si could be used to implement this strategy. In our previous work, the dynamic range for a sandwich fluorescence immunoassay for TNF- $\alpha$ was from $20 \mathrm{pg} \mathrm{mL}^{-1}$ to $\sim 50 \mathrm{ng} \mathrm{mL}^{-1}$, and we suggest that the upper limit of the dynamic range could be increased to $1 \mu \mathrm{M}$ using the method described here.

\section{Conclusions}

Microfluidic networks offer several advantages over conventional microtiter plates where the assays are done using much larger volumes. However, to take full advantage of microfluidic networks, it is important to identify the major parameters of assays in microfluidics as well as their interplay. The theoretical model developed here aims at understanding the relative importance of the transport of analyte in a microchannel by diffusion and convection, the binding kinetics between an analyte in solution and a surface-immobilized antibody, and the footprint of a capture antibody on the substrate for the assay. The flow velocity of the analyte solution in the microchannel is probably the parameter that is the most practical to vary using microfluidics with capillary pumps or using forced evaporation. Strategies to increase the surface density and orient the capture antibodies can also be applied to enhance the performances of the assays greatly. The substrate for the assay surface could be roughened, for example, or capture antibodies could be immobilized via their Fc part with cross-linkers or protein A or G, or using affinity-contact printing (Bernard et al., 2001a). Many assays would benefit from using very small amounts of sample or from being very fast. As the overall number of analyte molecules in miniaturized assays is typically small ( $\leq 1$ femtomol), it is important to exploit analytes as much as possible. An exciting outcome of this model is the indication that an analyte could be detected with picomolar sensitivity with a capture step of only 2 min duration using appropriately engineered capture sites. Conversely, longer assays and low flow rates might be used to perform $1 \mathrm{pM}$ sensitivity assays using a few tens of nanoliters only. The model presented here and the experimental results obtained previously coincide in showing that microfluidic networks have an excellent potential to miniaturize surface immunoassays that have demanding performances, such as in point-of-care testing where multiple disease markers could be detected at low concentrations in a single drop of sample and within minutes.

\section{Appendix: Supplementary Information}

\section{A. Implementation of the model}

The model was implemented using the method described in Griebel et al. (1995) and is divided in two parts: the first part calculates the velocity vector field and the second calculates the convection-diffusionequation and the binding kinetics to the concentration field.

The velocity vector field is calculated using the finite difference form of the Navier-Stokes equation. The general impulse equations

$$
\begin{aligned}
\frac{\partial u}{\partial t}+\frac{\partial p}{\partial x}= & \frac{1}{R e}\left(\frac{\partial^{2} u}{\partial x^{2}}+\frac{\partial^{2} u}{\partial y^{2}}\right) \\
& -\frac{\partial\left(u^{2}\right)}{\partial x}-\frac{\partial(u v)}{\partial y} \\
\frac{\partial u}{\partial t}+\frac{\partial p}{\partial y}= & \frac{1}{R e}\left(\frac{\partial^{2} v}{\partial x^{2}}+\frac{\partial^{2} v}{\partial y^{2}}\right) \\
& -\frac{\partial(u v)}{\partial x}-\frac{\partial\left(v^{2}\right)}{\partial y}
\end{aligned}
$$

are discretized on a regular staggered grid, Figure 9. To prevent numerical oscillations the different variables for velocity in the $x$ - and $y$-direction, $u, v$, and the pressure $P$ are localized at diverse positions on the grid cells. The boundary conditions are "no-slip" $(u=0)$ on the channel walls, "inflow" ( $u=$ const. $)$ at the inlet and "outflow" $\left(\frac{\partial(u, v)}{d n}=0\right)$ at the channel end. Furthermore we have to prevent stability problems occurring in discretized secondorder differential equations at convection-dominated flow conditions. For this, a variety of different methods are described in literature. From these methods, we chose the

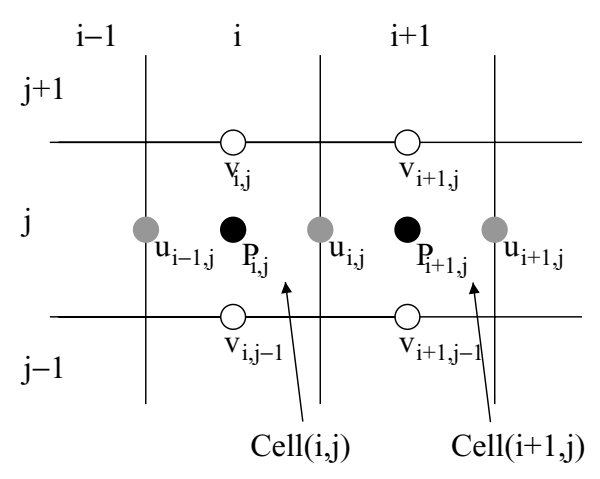

Fig. 9. 2D staggered grid used to model the flow by diffusion and convection. 
donor-cell scheme (Gentry et al., 1966):

$$
\begin{aligned}
{\left[\frac{d(k u)}{d x}\right]=} & \frac{1}{2 \delta x}\left(k_{r}\left(u_{i}+u_{i+1}\right)\right. \\
& \left.-k_{l}\left(u_{i-1}+u_{i}\right)\right)+\frac{1}{2 \delta x} \\
& \left(\left|k_{r}\right|\left(u_{i}-u_{i+1}\right)-\left|k_{l}\right|\left(u_{i-1}-u_{i}\right)\right),
\end{aligned}
$$

which has a good stability performance and combined it with the central difference scheme (Hirt et al., 1975):

$$
\left[\frac{d u^{2}}{d x}\right]_{i}=\frac{1}{\delta x}\left(\left(\frac{u_{i}+u_{i+1}}{2}\right)^{2}-\left(\frac{u_{i-1}+u_{i}}{2}\right)^{2}\right),
$$

which has a higher approximation performance than the donor-cell scheme. Equations (10) and (11) are shown as a one-dimensional example. They are averaged using a real parameter $\gamma$ chosen from the interval $[0,1]$ :

$$
\gamma \cdot \text { Donor Cell }+(1-\gamma) \cdot \text { Central Difference. }
$$

We have to introduce another stability condition for the time step to guaranty the stability of the numerical algorithm. We use a time-step control which includes the Courant-Friedrichs-Levi conditions:

$$
\begin{aligned}
& \delta t=\tau \min \left(\frac{\operatorname{Re}}{2}\left(\frac{1}{\delta x^{2}}+\frac{1}{\delta y^{2}}\right)^{-1},\right.\left.\frac{\delta x}{\left|u_{\max }\right|}, \frac{\delta y}{\left|v_{\max }\right|}\right) \\
&\tau \in] 0,1]
\end{aligned}
$$

These conditions prevent volume elements of liquid from moving further than one cell width, $\delta x$ or $\delta y$, during the time $\delta t$.

The second part of the model applies only the convection-diffusion equation, equation (3), and the binding kinetics, equation (4), to the concentration field. These equations have been discretized using the same models. The boundary conditions for equation (3) are a "constant concentration" at the inlet, "no flow" across the channel wall, and a simple "outflow" condition. For boundary cells next to the capture area, equation (4) is linked to equation (3) via the source term which can reduce or increase the analyte concentration in this boundary cell depending on the saturation of the capture area and the binding constants.

\section{B. Validation of the implemented numerical model}

Specific problems that can be described by analytical solutions are compared with the simulated results. Figure 10 shows the validation of the diffusion of the analyte during the assay. In this case, the flow velocity of the liquid in the microchannel is reduced to a minimal value to suppress convection. The mass transport then results from diffusion. The analytical solution is given as a reference. The correlation between the numerical and the ana-

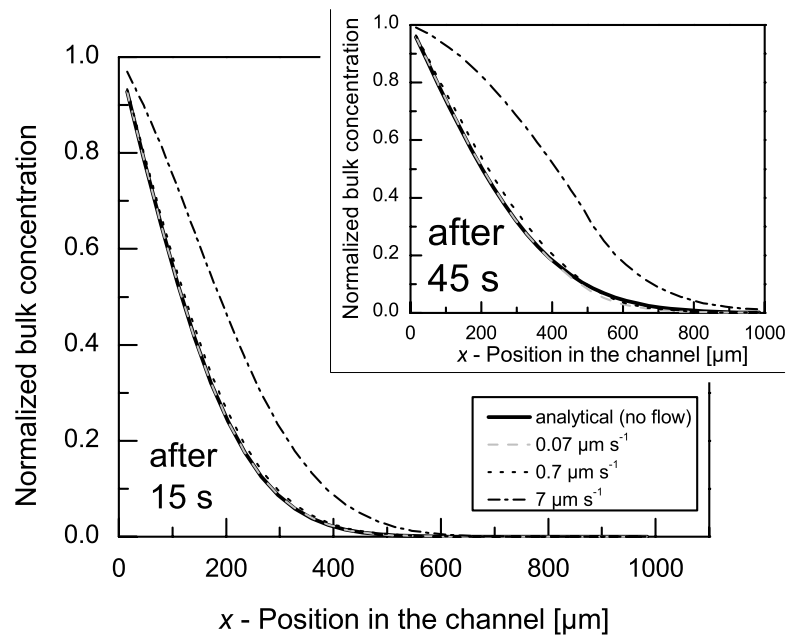

Fig. 10. Verification of the diffusion model using a slow convection in the $x$-direction. The graphs show the concentration profile along the channel after 15 and $45 \mathrm{~s}$, starting from infinite source at inlet $x=0 \mu \mathrm{m}$ into the initially empty channel. At flow velocities $\leq 0.07 \mu \mathrm{m} \mathrm{s}^{-1}$, diffusion predominates mass transport. Convection starts to have an effect on the transport of analyte in the microchannel for a flow velocity of $0.7 \mu \mathrm{m} \mathrm{s}^{-1}$, and substantially contributes to the transport for velocities $>7 \mu \mathrm{m} \mathrm{s}^{-1}$.

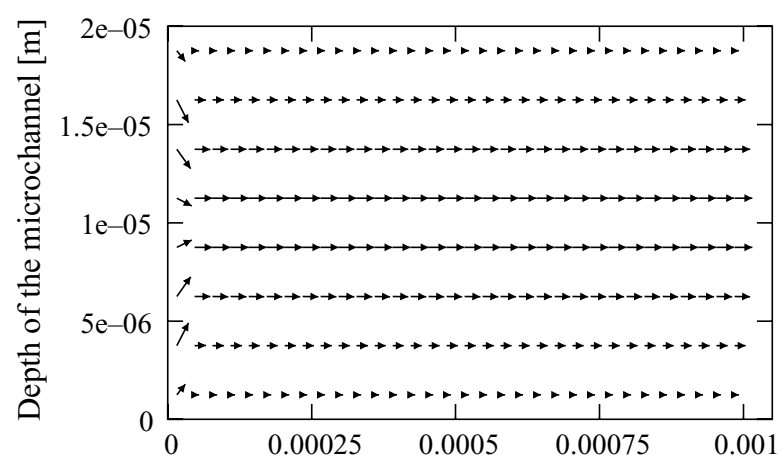

(a) $\quad x$-Position in the channel [m]

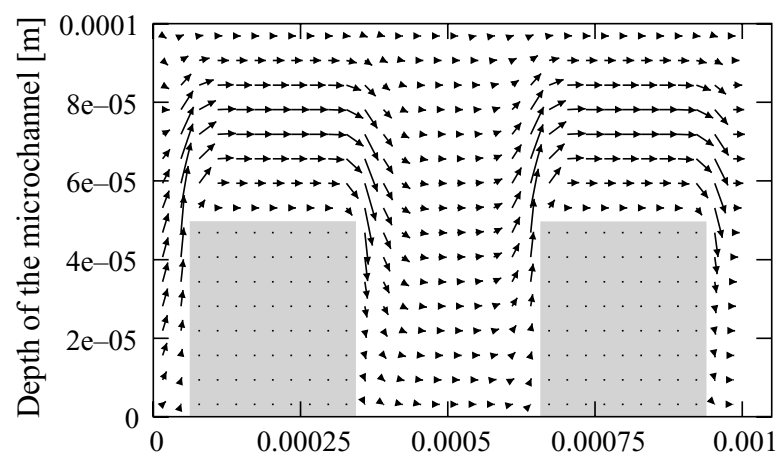

(b)

$x-$ Position in the channel [m]

Fig. 11. Visualization of the self-established laminar flow profile for (a) a short zone of flow development (at the inlet on the left-hand side) where the boundary conditions are "constant inflow" (left), "no-slip" (top, bottom) and "outflow" (right), and (b) for a channel having two obstacles. 


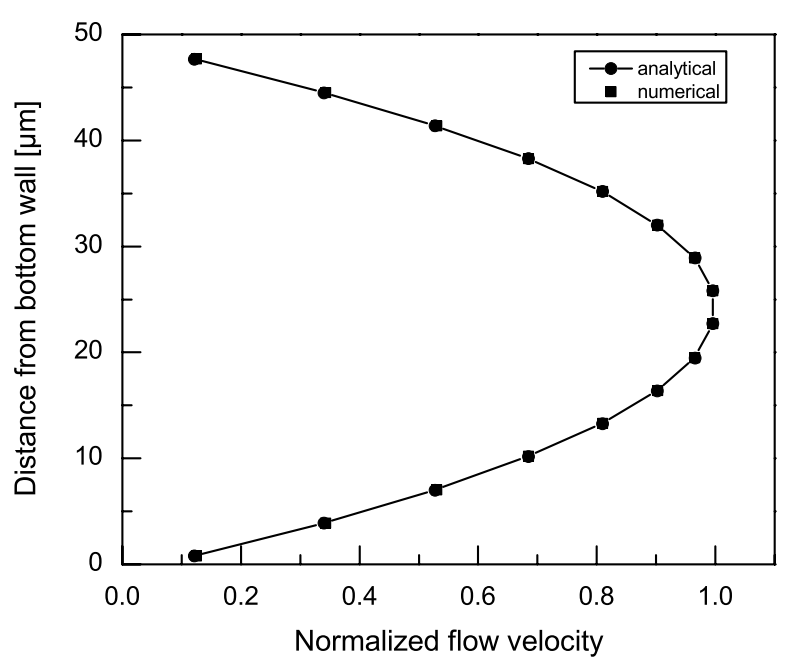

Fig. 12. The resemblance of the flow profiles obtained with the analytical and numerical model validates the numerical model describing the velocity flow profile. The flow profile here occurs at a $x$-position outside the flow development zone for a $50 \mu \mathrm{m}$ deep channel. The lines are provided as guides to the eye.

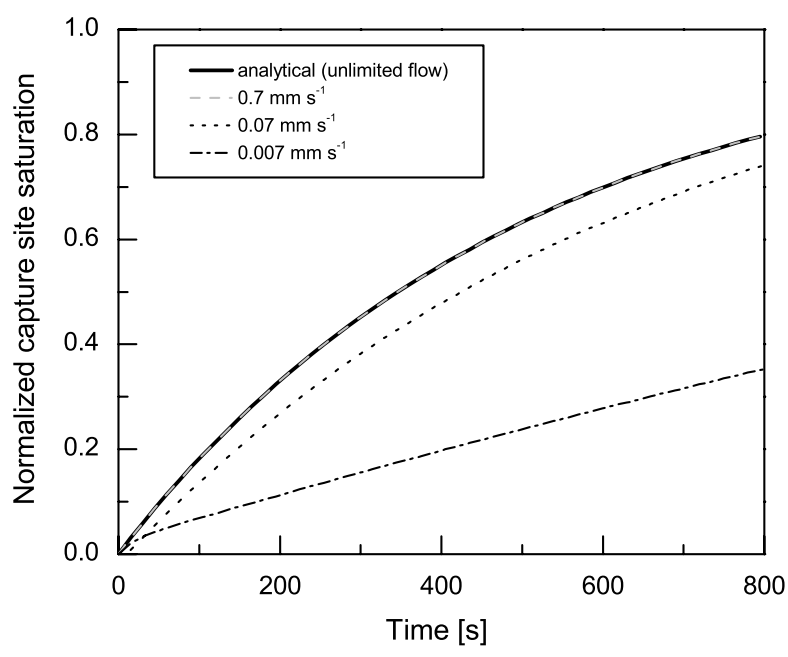

Fig. 13. Saturation of immobilized capture sites on a channel wall segment at $1 \mathrm{nM}$ analyte concentration. The binding reaction, represented by a ligand receptor model, is not slowed down at flow velocities of $0.7 \mathrm{~mm} \mathrm{~s}^{-1}$.

lytical solutions is excellent for the two graphs shown in Figure 10.

The flow velocity and the parabolic profile also have an important effect on the mass transport of analyte within the microchannel. Figure 11 gives an example of the capabilities of the model to describe the flow in the microchannel. Figure 12 shows the agreement between the analytical and the numerical solution describing the selfestablished flow profile.

The correctness of the ligand-receptor binding model is shown in Figure 13. No significant analyte exploitation occurs at high flow velocities. The curves describing the saturation of the binding sites obtained with the numerical and the analytical model are in agreement.

\section{Acknowledgments}

M. Z. acknowledges financial support from the Swiss Commission for Technology and Innovation (KTI, 7039.1 TNS) and M. W. from Nanotechnology of the Swiss National Science Foundation (NCCR). We thank H. J. Güntherodt, G. Dernick, H. Schmid and D. Juncker for helpful discussions.

\section{References}

L.M. Amzel and R.J. Poljak, Annu. Rev. Biochem. 48, 961-997 (1979).

F.S. Apple, R.H. Christenson, R. Valdes, A. Andriak, A. Berg, S. Duh, Y. Feng, S.A. Jortani, N.A. Johnson, B. Koplen, K. Mascotti, and A.H.B. Wu, Clin. Chem. 45, 199-205 (1999).

G. Balgi, D.E. Leckband, and J.M. Nitsche, Biophys. J. 68, 2251-2260 (1995).

A. Bernard, D. Fitzli, P. Sonderegger, E. Delamarche, B. Michel, H.R. Bosshard, and H. Biebuyck, Nature Biotechnology 19, 866-869 (2001a).

A. Bernard, B. Michel, and E. Delamarche, Anal. Chem. 73, 8-12 (2001b).

S. Cesaro-Tadic, G. Dernick, D. Juncker, G. Buurman, H. Kropshofer, B. Michel, C. Fattiger, and E. Delamarche, Lab on a Chip 4, 563-569 (2004).

E. Delamarche, in Nanobiotechnology, edited by C. Niemeyer and C. Mirkin (Wiley-VCH Verlag, Weinheim, 2004), pp. 31-52.

D.A. Edwards, J. Appl. Math. 63, 89-112 (1999).

D.A. Edwards, B. Goldstein, and D.S. Cohen, J. Math. Biol. 39, 533-561 (1999).

R.P. Ekins, Clin. Chem. 44, 2015-2030 (1998).

Genetix, 'aQuire microarray scanner specification sheet'. Genetix Ltd., http://www.genetix.com (2004).

R. Gentry, R. Martin, and B. Daly, J. Comp. Phys. 1, 87-118 (1966).

M. Griebel, T. Dornseifer, and T. Neunhoeffer, Numerische Simulation in der Strömungsmechanik (Vieweg, Braunschweig/Wiesbaden, 1995).

C. Hirt, B. Nichols, and N. Romero, Technical Report LA-5852, Los Alamos Scientific Lab. (1975).

J. Jenkins, B. Prabhakarpandian, K. Lenghaus, J. Hickman, and S. Sundaram, Anal. Biochem. 331, 207-215 (2004).

D. Juncker, H. Schmid, U. Drechsler, H. Wolf, M. Wolf, B. Michel, N. de Rooij, and E. Delamarche, Anal. Chem. 74, 6139-6144 (2002).

W. Koenig, M. Sund, B. Filipiak, A. Döring, H. Löwel, and E. Ernst, Arterioscler. Thromb. Vasc. Biol. 18, 768-772 (1998).

A. Manz and H. Becker, Microsystem Technology in Chemistry and Life Science (Springer-Verlag, Heidelberg, 1998).

S. Metsämuuronen, S. Reinikainen, and M. Nyström, Desalination 149, 453-458 (2002).

D.G. Myszka, X. He, M. Dembo, T.A. Morton, and B. Goldstein, Biophys. J. 75, 583-594 (1998).

N.T. Nguyen and S.T. Wereley, Fundamentals and Applications of Microfluidics (Artech House, Boston, 2002).

K.L. Prime and G.M. Whitesides, Science 252, 1164-1167 (1991). 
L.C. Santora, Z. Kaymkcalan, P. Sakorafas, I.S. Krull, and K. Grant, Anal. Chem. 299, 119-129 (2001).

K.E. Sapsford, Z. Liron, Y.S. Shubin, and F.S. Ligler, Anal. Chem. 73, 5518-5524 (2001)

E.F.H. Tay (ed.), Microfluidics and BioMEMS Applications (Kluwer Academic Publishers, Boston, 2002).
R. Vijayendran, F.S. Ligler, and D.E. Leckband, Anal. Chem. 71, 54055412 (1999).

D. Wild (ed.), The Immunoassay Handbook (Nature Publishing Group, London, 2001).

M. Wolf, D. Juncker, B. Michel, P. Hunziker, and E. Delamarche. Biosens. Bioelectr. 19, 1193-1202 (2004). 